\title{
Factors Associated with Duration of Overall Treatment Time for Cervical Cancer Treated with Definitive Chemoradiotherapy
}

Vladimir Valakh $^{1}$, Bryan C. Coopey ${ }^{1}$

1. Radiation Oncology, Allegheny Health Network Cancer Institute, Pittsburgh, USA

$\square$ Corresponding author: Vladimir Valakh, vladimir.valakh@ahn.org

\section{Abstract}

Introduction

For women with intact cervical cancer treated by definitive chemoradiotherapy, the adverse impact of treatment prolongation is well-established. We aimed to identify potentially modifiable factors associated with the unwanted increase in the time required to complete the prescribed course of therapy.

Methods

We retrospectively examined treatment records of 104 consecutive cervical cancer patients receiving chemoradiation for cervical cancer, which included cervical high-dose-rate intracavitary brachytherapy performed at a single referral center. Association of factors, including distance to care, driving time, and income level with overall treatment time, was explored.

Results

Guideline-concordant treatment duration was achieved in 34\% of cases. There was no significant correlation between treatment duration and any of the patient-related characteristics assessed in this study. Transitioning of the radiation oncology physician staffing at the brachytherapy center from a private practice non-integrated group to a large integrated group was associated with a decrease in mean overall treatment time, 61 vs. 68 days, p =0.007.

\section{Conclusions}

We did not identify a modifiable patient-related factor amenable to a financial intervention. Administration of brachytherapy within an integrated system resulted in a shortened duration of the entire course of therapy for intact cervical cancer.

Categories: Obstetrics/Gynecology, Oncology, Radiation Oncology

Keywords: completion, distance to care, cervical cancer, overall treatment time, chemoradiotherapy, brachytherapy, implant, driving, integrated, high-dose-rate

\section{Introduction}

Cervical carcinoma is a major healthcare burden with approximately 13,000 cases diagnosed 
annually in the United States (US) [1]. Disproportionally, this disease affects underserved populations which lack the ready access to the preventive gynecologic care [2]. For Stages IB2IVA cervical cancer, the most common treatment entails definitive chemoradiotherapy (CRT): the combination of fractionated external beam radiotherapy (EBRT), concurrent weekly chemotherapy, and high-dose-rate intracavitary brachytherapy [3]. Retrospective data point to the adverse impact of CRT prolongation on cancer control, with the most commonly used cutoff of eight weeks [4-5]. However, the patterns of care studies have consistently revealed that < $50 \%$ of women complete CRT within the intended time interval [6-8].

Reasons for CRT prolongation have not been conclusively established. Most research on the subject was carried out before the implementation of concurrent chemotherapy or outpatient brachytherapy. In the modern era, association with increased time to completion of CRT was shown for such disparate factors, such as patient age, development of side effects of CRT, lack of focused patient education about brachytherapy, and delay to perform the first implant [8-10].

A notable feature of definitive CRT for cervical cancer is a large number of therapeutic sessions. A commonly used schedule calls for 25 daily consecutive fractions of EBRT and five bi-weekly implants started towards the end of external beam irradiation and performed separately from EBRT and chemotherapy days. Additional appointments for ancillary testing and procedures, such as repeat diagnostic imaging, EBRT boost, and the temporary insertion of a brachytherapy sleeve, are also needed [11]. The successful delivery of this intense treatment package requires the commitment of substantial financial and temporal resources by the patients and their caregivers.

Our facility has been serving as a cervical implant referral center for a large geographic region which included designated economically distressed areas [12]. We have observed that patients residing in those areas, who also were traveling long distances to receive brachytherapy, were at risk of CRT prolongation through delay in starting and completing implants. We hypothesized that a population existed for which the cost of travel and lodging for brachytherapy constituted a barrier to care. This represented a potential target for a focused financial intervention to improve the quality of healthcare delivery. Therefore, we carried out a single-institutional retrospective study to identify factors associated with the duration of overall treatment time in intact cervical cancer.

\section{Materials And Methods}

The study was approved by the Allegheny-Singer Research Institute-West Penn Allegheny Health System (ASRI-WPAHS) Institutional Review Board. One hundred and four consecutive patients who received curative brachytherapy to the intact cervix from 2008 - 2017 at a single hospital-based radiation oncology center were identified. The 2018 Federation of Gynecology and Obstetrics clinical stages ranged from IB1 to IVA [13]. The median age was 55 years (range: $25-90)$.

All patients were treated uniformly, receiving 45 - 50 Gy of EBRT to the pelvis in 25 fractions, concurrent weekly cisplatin chemotherapy, and intracavitary high-dose-rate brachytherapy boost in five sessions, given as an outpatient. EBRT and chemotherapy were administered at our center in $28 \%$ of women, while $72 \%$ were treated by local oncologists in one of 19 referring centers. For EBRT, either intensity-modulated radiotherapy planning and delivery technique or three-dimensional (3D) conformal radiotherapy was used at the discretion of the radiation oncologists. All brachytherapy was administered at our facility. Insertion of either tandem and ovoids, tandem and ring, or tandem and cylinder (< $10 \%$ of cases) apparatuses were facilitated by the temporary placement of a cervical sleeve in the operating room at our hospital [11]. Sleeve insertion surgery was performed either before commencing brachytherapy or on the day of the first implant, per a case-by-case consideration. Fractional brachytherapy doses ranged 
from 5 to 6 Gy. The delivery of the EBRT boost, when needed (for $<10 \%$ of patients), was interdigitated with brachytherapy. The duration of the overall treatment time was calculated as the number of calendar days from the first EBRT fraction to the last implant. Women receiving interstitial brachytherapy were excluded from this study, as were those patients unable to complete five sessions of brachytherapy.

In mid-2011, radiation oncology physician staffing at our center was transitioned from a nonintegrated private practice group to a large integrated group [14]. Thus, 56\% of patients in this study have received brachytherapy within an integrated delivery care system, while $44 \%$ have not.

In our brachytherapy practice, $>90 \%$ of patients utilized a private car mode of transportation. Therefore, a key independent variable in this study was the one-way driving distance from the patient's current residence to the brachytherapy center, calculated using web-based software [15]. Similarly, we recorded expected one-way driving time for each patient, taking into account the typical rush-hour delays for a weekday morning arrival at our center. In addition, the geographical distance to brachytherapy was recorded using the straight-line method [16]. Income level was assessed using the 2015 US Census tract median household income data [17]. The rural versus urban residence distinction using rural-urban continuum codes was not deemed feasible for this study as a large county at the center of our geographic region was composed of the metropolitan and non-metropolitan areas [18]. For this study purpose, brachytherapy during the holiday season, when our center had scheduled days off, was defined as the receipt of at least one implant from the fourth week of November through the first week of January.

Associations between variables were assessed by examining scatter plots and performing Pearson correlation. The means for subgroups were compared using the independent variables T-test. Fisher's exact test was used to evaluate the distribution of categorical variables. The significance level was set at $\mathrm{p}=0.05$. A multiple linear regression model using two independent variables was created and reviewed. Statistical analysis was performed using the IBM Statistical Package for Social Sciences (SPSS), version 23, software (IBM Corp., Armonk, NY).

\section{Results}

Patient and treatment characteristics are listed in Table 1. The median duration of CRT was 60 days. Thirty-four percent of patients completed CRT within eight weeks of the treatment start date and $56.7 \%$ finished within nine weeks. The average one-way driving time to brachytherapy exceeded one hour. The median household income of the study population was $83 \%$ of the national median. 


\section{Cureus}

\section{Parameter}

Median age, years

EBRT and brachytherapy at different centers, $\mathrm{N}$

Brachytherapy within integrated system, $\mathrm{N}$

Median straight line distance to brachytherapy, miles

Median driving distance, miles

Median driving time, minutes

Brachytherapy during the holiday season, $\mathrm{N}$

Median household income, US dollars

Median treatment duration, days
55

$75(72 \%)$

$58(56 \%)$

24

32

69

$15(14 \%)$

46,700

60
$43-68$

$\mathrm{N} / \mathrm{A}$

$\mathrm{N} / \mathrm{A}$

$12-41$

$16-58$

$50-92$

$\mathrm{N} / \mathrm{A}$

$41,000-56,700$

$55-70$

\section{TABLE 1: Patient and Treatment Characteristics}

EBRT: external beam radiotherapy; N: number; N/A: not applicable; US: United States

No significant correlation was demonstrated between the treatment duration and either the distance to care, the driving time, or the income level (Figures 1-3). For the patients in the lower quartile for the distance to care, the duration of CRT was statistically significantly shorter in the univariate analysis (Table 2). No association between the level of income and the short distance to care was observed to explain this observation $(\mathrm{p}=0.44)$. Patients living farther away from our center were more likely to receive EBRT and chemotherapy before brachytherapy in the community instead of our center $(\mathrm{p}<0.001)$. However, no influence of the location of the oncology center tasked with EBRT and chemotherapy delivery on the overall treatment duration was present $(p=0.27)$. Notably, women with the farthest distance to brachytherapy or those in the lower quartile for income were not more likely to experience CRT prolongation compared to the rest of the study population (Table 2). 


\section{Cureus}

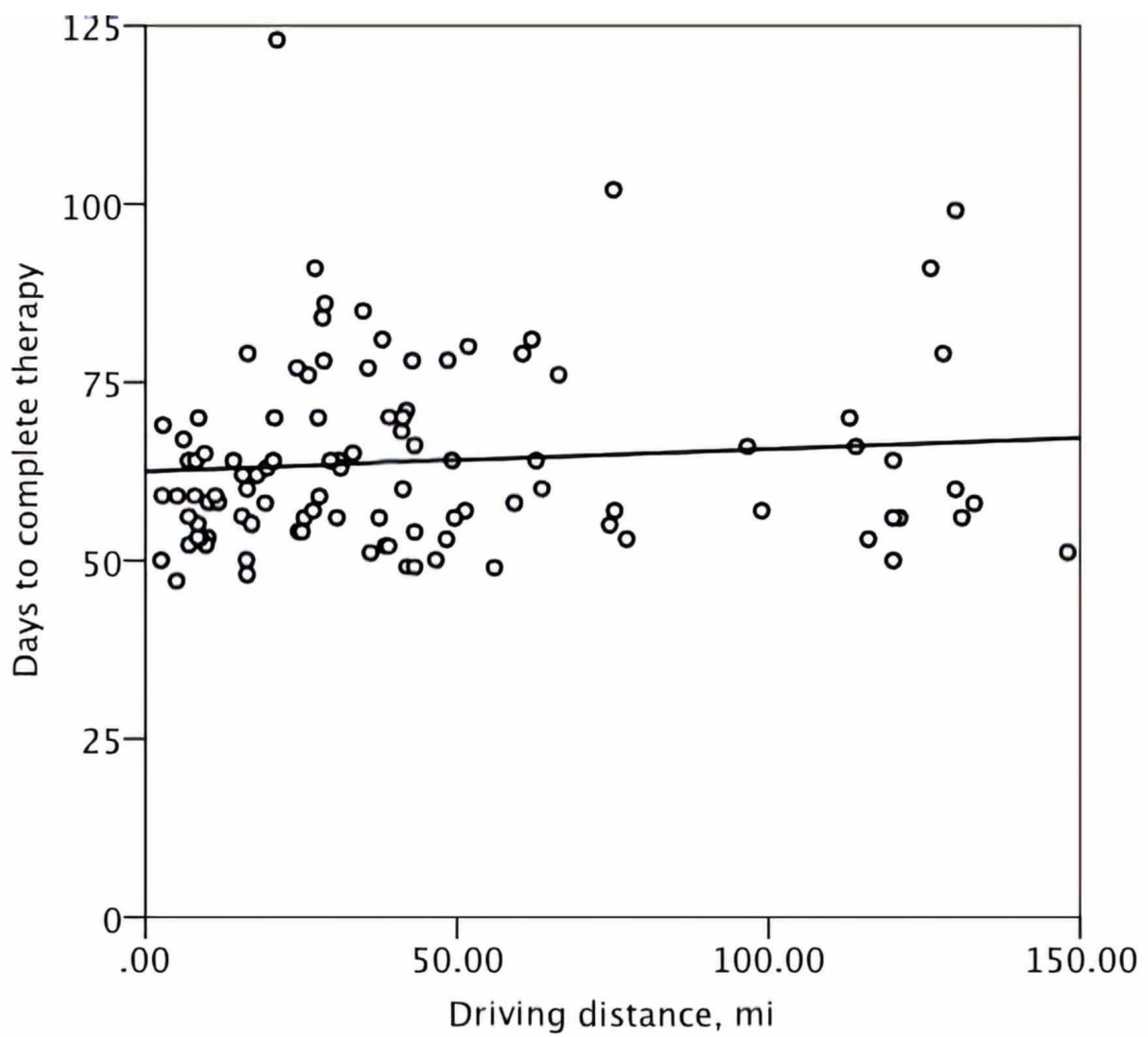

FIGURE 1: A scatter plot demonstrating the distribution of the treatment duration and one-way driving distance in miles (mi)

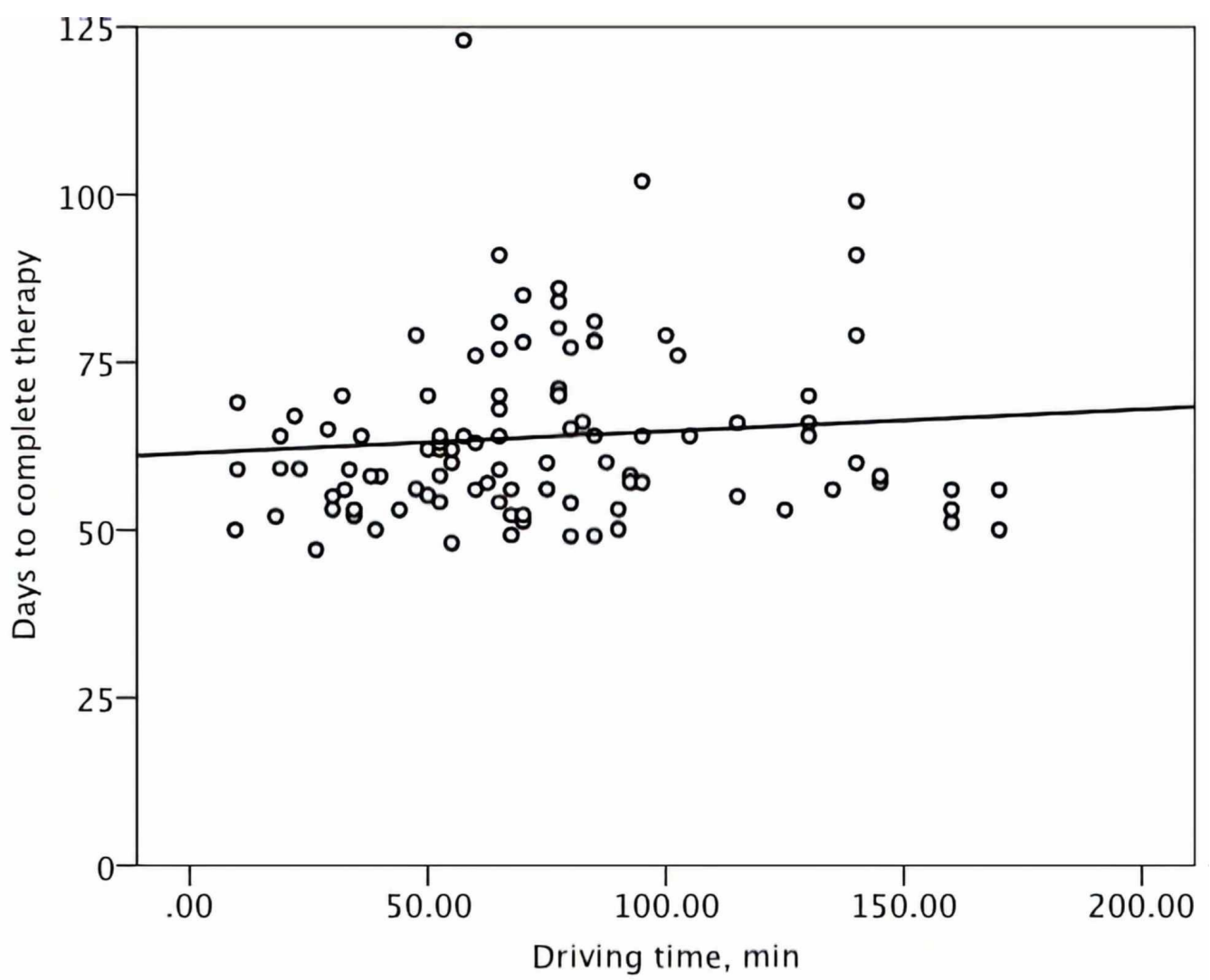




\section{Cureus}

FIGURE 2: A scatter plot demonstrating the distribution of the treatment duration and one-way driving time to the brachytherapy center in minutes (min)

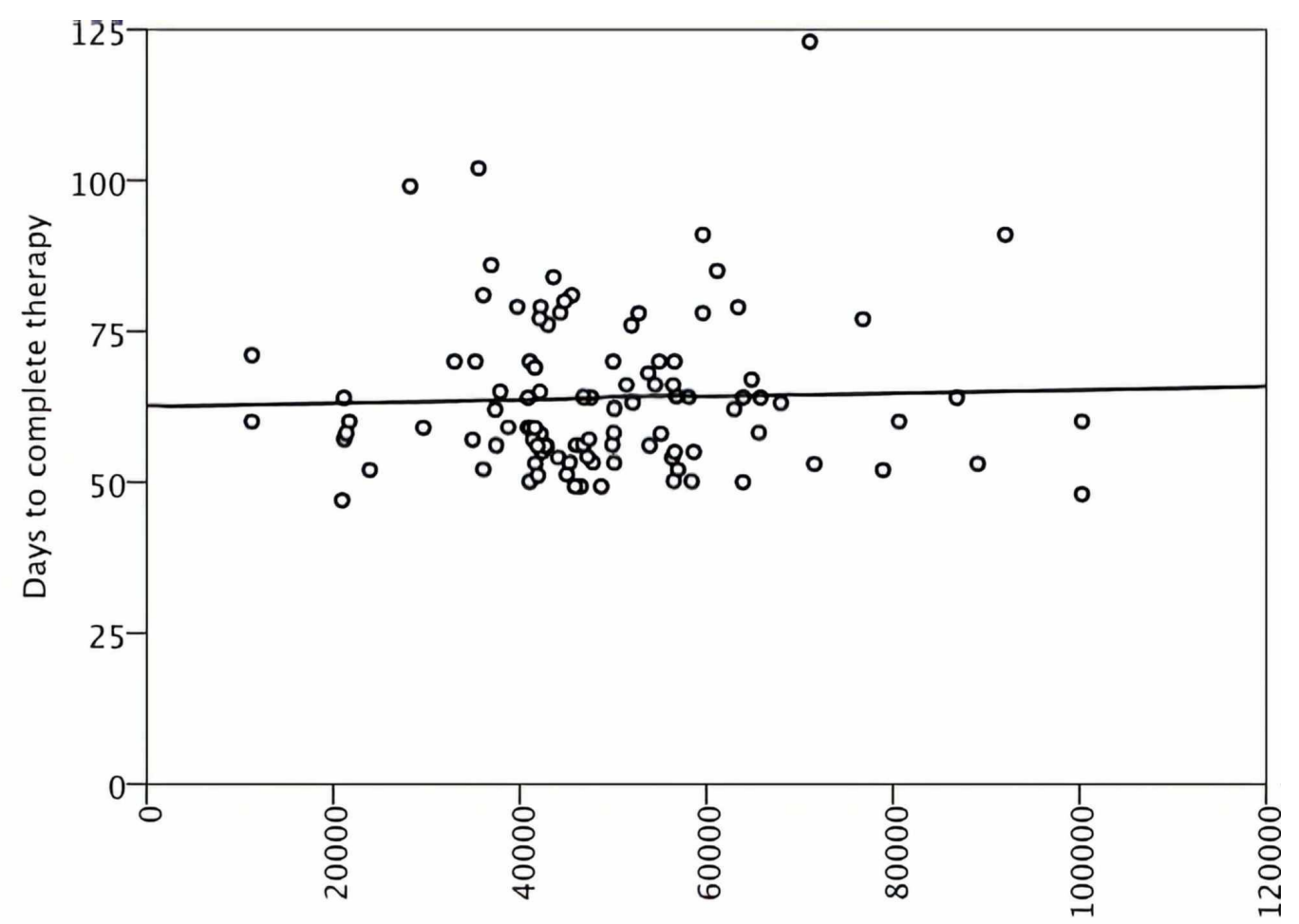

Annual income, $\$$

FIGURE 3: A scatter plot demonstrating the distribution of duration of overall treatment time according to the estimated annual income

\$: United States dollars

\section{Parameter} $>$
55

Age, years

Geographical distance to brachytherapy,

$\leq$

EBRT at a different center 24
Mean radiotherapy duration, days 65 $\begin{array}{ll}\leq & 63\end{array}$ Yes 65 No 62
Univariate $p$ - Multivariate $p$ value value 


\section{Cureus}

miles

0.68

63

Driving distance, miles

Driving distance, miles

Driving time, minutes

Driving time, minutes

$\leq$

Brachytherapy during the holiday season

\section{TABLE 2: Association of Study Variables with Overall Treatment Time}

EBRT: external beam radiotherapy

The administration of brachytherapy within an integrated delivery care system was associated with a shorter duration of overall treatment time (Table 2). Forty-one percent of patients treated in that environment completed CRT within eight weeks vs. $26 \%$ who did so before the transition to the integrated system $(\mathrm{p}=0.077)$. In the multivariate analysis, integrated care maintained the association with faster treatment completion while distance did not (Table 2).

\section{Discussion}

This single-institutional retrospective study has demonstrated that the minority of patients with carcinoma of the intact cervix have completed definitive chemoradiotherapy within the timeframe suggested by the current guidelines. Sixty-six percent of women required longer 
than eight weeks to receive the prescribed therapy, which is similar to the recent large database studies on the modern cervical CRT in the US [7-8]. CRT starts with the first fraction of EBRT and concludes on the day of the last implant. Therefore, prolongation of CRT can occur due to breaks in EBRT, inability to initiate brachytherapy sufficiently early in the treatment program, delays in completion of brachytherapy, or any combination of these three factors.

As most women in this study received EBRT and chemotherapy in a community setting, the precise treatment calendars and the toxicity descriptions could not be collected. However, when those data were available, we have noted that unscheduled EBRT breaks were rare. High-grade treatment side effects after completing EBRT and cisplatin were uncommon as well. It is in line with data demonstrating an excellent tolerance of 45 - $50 \mathrm{~Gy}$ of external beam radiotherapy with intravenous cisplatin chemotherapy [19]. Thus, for women with prolonged overall treatment time, non-receipt of timely brachytherapy was the most likely reason.

Based on case observations, we have postulated that patient-related factors were the underlying mechanism of delay in starting and completing brachytherapy. Specifically, we have hypothesized that for a subgroup of patients, the cost of private automobile travel and the overnight lodging for five outpatient brachytherapy sessions constituted a modifiable barrier to care. However, this study demonstrated that overall treatment time was not associated with either driving distance, time, or income level. A similar observation has been reported by Spees et al. [8]. Therefore, a focused financial intervention, such as the creation of an institutional fund to pay for cervical patients' gasoline and lodging expenses, was not likely to improve the overall treatment time for intact cervical cancer.

Instead, the results of this study suggest physician and program-related factors for non-receipt of timely brachytherapy and, in turn, lengthening of the overall treatment time. Compared to other forms of radiotherapy, cervical brachytherapy has been reported to require a much larger commitment of the physician's time effort [20]. Further, guideline-concordant brachytherapy for cervical cancer patients who start EBRT and chemotherapy at a different center, as it was done for most of the women in our study, necessitates additional resources for coordinating care between two treating teams. Improved coordination is a feature of integrated healthcare delivery systems [21]. We observed improvement in overall treatment time after transitioning physician staffing to an integrated group.

Lastly, the optimal number of total therapeutic sessions for intact cervical cancer treated with external beam radiotherapy, concurrent chemotherapy, and high-dose brachytherapy may be potentially decreased without affecting treatment efficacy. Supported by the findings of this study, from 2017 onwards, we have aimed to reduce the typical number of intended EBRT fractions and implant insertions from 25 to 22 and from five to four, respectively, while maintaining the same biologically equivalent prescribed radiotherapy dose [22]. That should shorten overall treatment time by one week in an uncomplicated case, potentially improving pelvic cancer control [5].

\section{Conclusions}

The duration of the overall treatment time in cervical cancer treated with definitive CRT constitutes an important measure of the quality of care. This retrospective single-institutional study demonstrated that a minority of patients completed CRT within a time interval suggested by the clinical guidelines. The most likely culprit was the inability to initiate and/or complete cervical brachytherapy in a timely manner. Interestingly, we were unable to identify a patientrelated factor for treatment prolongation, which could be addressed through a financial intervention, such as a travel and lodging fund. Instead, performing brachytherapy within an integrated care network was associated with faster completion of CRT for women with cervical cancer. We have concluded that while patients' cooperation is a prerequisite, improvement in 
overall treatment time in cervical cancer requires a concerted effort by the physicians and the ancillary healthcare staff.

\section{Additional Information \\ Disclosures}

Human subjects: Consent was obtained by all participants in this study. Allegheny-Singer Research Institute-West Penn Allegheny Health System (ASRI-WPAHS) Institutional Review Board issued approval 218-293. November 16, 2018 Vladimir Valakh, MD Department of Radiation Oncology RE: 2018-293 "Identification of factors associated with prolongation of the overall treatment time for cervical cancer treated with chemoradiotherapy without surgery" Dear Dr. Valakh: The ASRI-WPAHS Institutional Review Board (IRB) is in receipt of the abovereferenced protocol. The IRB has reviewed the information and has determined that the abovereferenced protocol is approved. The following waivers were also approved: Waiver of HIPAA Authorization Waiver of Informed Consent This protocol has been reviewed via the "expedited review” process (Expedited Category 5 - Research involving materials (data, documents, records, or specimens) that have been collected, or will be collected solely for non-research purposes (such as medical treatment or diagnosis) and approved in accordance with Institutional, Federal and State regulations by the IRB. It is the responsibility of the investigator to obtain any other necessary approvals prior to implementation of the research (AGH and/or ASRI). Your approved protocol will be subject to review within one year from the date of initial review by the IRB. Approved: November 16, 2018 Approval Period Expires: November 15, 2019 Please be aware of the record-keeping responsibilities involved in your protocol. Sincerely, Farrel Buchinsky, MBChB, BSc(Hons)(Med), FACS Vice-Chairman Institutional Review Board. Animal subjects: All authors have confirmed that this study did not involve animal subjects or tissue. Conflicts of interest: In compliance with the ICMJE uniform disclosure form, all authors declare the following: Payment/services info: All authors have declared that no financial support was received from any organization for the submitted work. Financial relationships: All authors have declared that they have no financial relationships at present or within the previous three years with any organizations that might have an interest in the submitted work. Other relationships: All authors have declared that there are no other relationships or activities that could appear to have influenced the submitted work.

\section{References}

1. Siegel RL, Miller KD, Jemal A: Cancer statistics, 2017. CA Cancer J Clin. 2017, 67:7-30. $10.3322 /$ caac. 21387

2. Gauri A, Messiah SE, Bouzoubaa LA, Moore KJ, Koru-Sengul T: Cervical cancer sociodemographic and diagnostic disparities in Florida: a population-based study (1981-2013) by stage at presentation. Ethn Health. 2018, Epub ahead of print:1-9. 10.1080/13557858.2018.1471669

3. Mayadev J, Viswanathan A, Liu Y, et al.: American Brachytherapy Task Group Report: a pooled analysis of clinical outcomes for high-dose-rate brachytherapy for cervical cancer. Brachytherapy. 2017, 16:22-43. 10.1016/j.brachy.2016.03.008

4. Song S, Rudra S, Hasselle MD, et al.: The effect of treatment time in locally advanced cervical cancer in the era of concurrent chemoradiotherapy. Cancer. 2013, 119:325-31. $10.1002 /$ cncr.27652

5. Mazeron R, Castelnau-Marchand P, Dumas I, et al.: Impact of treatment time and dose escalation on local control in locally advanced cervical cancer treated by chemoradiation and image-guided pulsed-dose rate adaptive brachytherapy. Radiother Oncol. 2015, 114:257-63. 10.1016/j.radonc.2014.11.045

6. Eifel PJ, Ho A, Khalid N, Erickson B, Owen J: Patterns of radiation therapy practice for patients treated for intact cervical cancer in 2005 to 2007: a quality research in radiation oncology study. Int J Radiat Oncol Biol Phys. 2014, 89:249-56. 10.1016/j.ijrobp.2013.11.228 
7. Smith GL, Jiang J, Giordano SH, Meyer LA, Eifel PJ: Trends in the quality of treatment for patients with intact cervical cancer in the United States, 1999 through 2011. Int J Radiat Oncol Biol Phys. 2015, 92:260-67. 10.1016/j.ijrobp.2015.01.037

8. Spees LP, Brewster WR, Varia MA, et al.: Examining urban and rural differences in how distance to care influences the initiation and completion of treatment among insured cervical cancer patients. Cancer Epidemiol Biomarkers Prev. 2019, 28:882-89. 10.1158/1055-9965.EPI18-0945

9. Eng T, Magel V: Reduction of brachytherapy treatment cancellation rates by systematic team approach. Universal J Management. 2013, 1:126-31.

10. Zaki M, Dominello M, Morris R, Miller S: Factors predictive of protracted course of radiation therapy in patients treated with definitive chemoradiation for cervical cancer. Cureus. 2016, 8:e558. 10.7759/cureus.558

11. Smit BJ, du Toit JP, Groenewald WA: An indwelling intrauterine tube to facilitate intracavitary radiotherapy of carcinoma of the cervix. Br J Radiol. 1989, 62:68-69. 10.1259/0007-1285-62733-68

12. Mark KP, Crosby RA, Vanderpool RC: Psychosocial correlates of ever having a Pap test and abnormal pap results in a sample of rural Appalachian women. J Rural Health. 2018, 34:14854. 10.1111/jrh.12265

13. Bhatla N, Berek JS, Cuello Fredes M, et al.: Revised FIGO staging for carcinoma of the cervix uteri. Int J Gynaecol Obstet. 2019, 145:129-35. 10.1002/ijgo.12749

14. Chen AB, Cronin A, Weeks JC, Chrischilles EA, Malin J, Hayman JA, Schrag D: Palliative radiation therapy practice in patients with metastatic non-small-cell lung cancer: a Cancer Care Outcomes Research and Surveillance Consortium (CanCORS) Study. J Clin Oncol. 2013, 31:558-64. 10.1200/JCO.2012.43.7954

15. Kelley KA, Young JI, Bassale S, et al.: Travel distance influences readmissions in colorectal cancer patients-what the primary operative team needs to know. J Surg Res. 2018, 227:220-27. 10.1016/j.jss.2018.02.022

16. Duncan DT, Castro MC, Blossom JC, Bennett GG, Gortmaker SL: Evaluation of the positional difference between two common geocoding methods. Geospat Health. 2011, 5:265-73.

10.4081/gh.2011.179

17. Selected Economic Characteristics. 2015: ACS 5-Year Estimates Data Profiles . (2016). Accessed: October 18, 2019: http://data.census.gov/cedsci/table?d=ACS\%205Year\%20Estimates\%20Data\%20Profiles \&table=DP03 \&tid=ACSDP5Y2015.DP03\&y=20....

18. Unger JM, Moseley A, Symington B, Chavez-MacGregor M, Ramsey SD, Hershman DL: Geographic distribution and survival outcomes for rural patients with cancer treated in clinical trials. JAMA Netw Open. 2018, 1:e181235. 10.1001/jamanetworkopen.2018.1235

19. Peters WA 3rd, Liu PY, Barrett RJ 2nd, et al.: Concurrent chemotherapy and pelvic radiation therapy compared with pelvic radiation therapy alone as adjuvant therapy after radical surgery in high-risk early-stage cancer of the cervix. J Clin Oncol. 2000, 18:1606-13. 10.1200/JCO.2000.18.8.1606

20. Bauer-Nilsen K, Hill C, Trifiletti DM, et al.: Evaluation of delivery costs for external beam radiation therapy and brachytherapy for locally advanced cervical cancer using time-driven activity-based costing. Int J Radiat Oncol Biol Phys. 2018, 100:88-94.

10.1016/j.ijrobp.2017.09.004

21. Wan TT, Lin BY, Ma A: Integration mechanisms and hospital efficiency in integrated health care delivery systems. J Med Syst. 2002, 26:127-43. 10.1023/A:1014805909707

22. Viswanathan AN, Beriwal S, De Los Santos JF, et al.: American Brachytherapy Society consensus guidelines for locally advanced carcinoma of the cervix. Part II: high-dose-rate brachytherapy. Brachytherapy. 2012, 11:47-52. 10.1016/j.brachy.2011.07.002 